\author{
Military Technical \\ College \\ Kobry El-Kobba \\ Cairo, Egypt
}

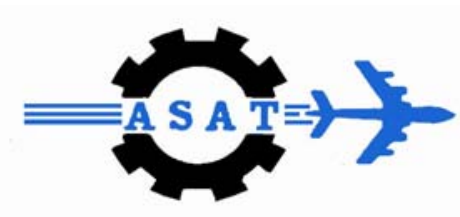

12-th International

Conference on

Aerospace Sciences \&

Aviation Technology

\title{
OPTIMIZATION OF SHAPE OF CONVERGENT-DIVERGENT NOZZLES BY USING IMPROVED SIMPLE GENETIC ALGORITHMS
}

\author{
Mazaheri $^{*}$ K., Khayatzadeh ${ }^{* *} \mathrm{P}$.
}

\begin{abstract}
Genetic Algorithms (GAs) have initiated a new scheme for solution of the complicated optimization problems. Here a genetic algorithm is developed for optimization of the convergent-divergent nozzles. First, a popular numerical method is chosen for solving the fluid dynamic problem. Then, optimization criterion and evaluation parameters (such as maximum thrust force) are determined. After introduction of the simple genetic algorithm, parameters of this method such as crossover probability, mutation probability and population size in each generation are used for optimization of the algorithm. After independent verification of the computational fluid dynamic scheme and the genetic algorithm, these two are integrated, and using different values for the above parameters, performance of the optimization algorithm for shape optimization of a nozzle is studied. Finally an optimum range of GA parameters are found, and are used to find optimum shape by less than $0.1 \%$ exhaustive search CPU time.
\end{abstract}

\section{KEYWORDS}

Genetic Algorithm, Mutation, Crossover, Elitism, Convergent-Divergent Nozzle.

\footnotetext{
* Professor of Aerospace Engineering Sharif University of Technology, P.O. Box 11365-5639, Tehran, Iran, Tel/Fax: (+9821) 66022731, mazaheri@sharif.edu

** Graduate Student of Aerospace Engineering Department, Sharif University of Technology, Tel: (+98912) 5806304, peyman khayatzadeh@yahoo.com
} 


\section{INTRODUCTION}

Genetic Algorithm (GA) is based on natural selection mechanism and natural genetics as a robust method among optimization techniques. Since for optimization, genetic algorithm uses information of objective function instead of derivative value and other useful information, it is different from the other optimization methods. In addition, genetic algorithm finds optimum point of a problem through a simultaneous multipoint search instead of a point by point search. These two properties of this algorithm cause genetic algorithm to be very useful and efficient in a wide range of engineering problems.

In recent years, genetic algorithm is combined with many computational fluid dynamic schemes and is used for optimization of aerodynamic shapes [1-3]. It is very attractive for design and optimization of aerodynamic shapes because unlike the gradient based methods, more likely it is able to find the global optimum point of an optimization problem [4]. However, its time complexity advantage is still on debate. Our main purpose here is to optimize the time complexity of the algorithm, so that it is more advantageous to gradient based methods in all aspects.

Optimization of an aerodynamic shape includes determining the values of design parameters. These values determine the geometric details of aerodynamic shapes, in a way that an objective function value is maximized while satisfying the aerodynamic constraints. Parameters of flow field must satisfy the governing equations of the flow field. Nowadays computational fluid dynamics (CFD) has reached to maturity, and we can efficiently solve the flow field equations for external and internal flows in all different subsonic, transonic and supersonic regimes in three different levels of simplifying assumptions as potential, Euler and Navier-Stokes equations. Our genetic algorithm is of course independent of solution method used in our CFD computations. In other words, any flow solution method could be integrated to any genetic algorithm optimization scheme [5,6]. This combination could appropriately be used for aerodynamic shape optimization. Design parameters are in fact parameters which determine the geometrical shape, and are due to satisfy the geometric constraints of the problem of finding a reasonable shape.

For example, in aerodynamic shape optimization of a wing, geometric parameters such as wing span, wing chord, wing twist angle, maximum chord thickness, radius of trailing edge and radius of leading edge ... in different cross sections of the wing must be limited to reasonable values.

In this paper, To find maximum thrust force of nozzle, shape of a convergent divergent nozzle is optimized by using a genetic algorithm. The convergent section of the nozzle is given by a second order polynomial with known coefficients. The divergent part of the nozzle is assumed to be a third order polynomial whose coefficients are determined by GA, under some geometrical constraints. Geometric constraints applied here are constant throat and exit areas of nozzle. Under these conditions, the coefficients of this third order polynomial may change in a special range to produce only reasonable shapes.

The nozzle flow is assumed quasi-one- dimensional. The thrust force of nozzle is obtained after numerical solution of the flow field by a finite volume method using Roe's flux approximation and a time marching (explicit) method [13]. Its verification is performed by comparison with the analytical solution of the nozzle flow. The genetic algorithm used is a simple genetic algorithm that has three operators namely reproduction, crossover, and mutation. This algorithm is explained in details in 
reference [7]. Its verification is also performed by finding the optimum point of a $10^{\text {th }}$ order polynomial in a specific range, and is compared with results in reference [7]. As the genetic algorithm is independent of the flow solution method (objective function) $[5,6]$, therefore it can be used for shape optimization of all kinds of convergent divergent nozzles to obtain maximum thrust force. The results, which are obtained by combination of the genetic algorithm and the numerical solution method for solving the internal flow of the nozzle, show the efficiency of the genetic algorithm in shape optimization of convergent divergent nozzles to find the maximum thrust force.

\section{GENETIC ALGORITHM}

Genetic algorithm (GA) is a mathematical algorithm which uses operational patterns of Darwin's principle in accordance to the survival of fitness and under basis of natural genetic processes such as mating, crossover, and mutation. Also it can change population of a single mathematical object (chromosome) with special fitness level to a new generation. Although genetic algorithms are considered as part of stochastic methods, they have simple deterministic processes. They apply information of the previous generation with a high efficiency to find new points. These algorithms are not completely random, and in the literature of genetic algorithm, a process usually can not be found that is completely random. This algorithm does not need to the derivative value of the objective function for optimization, and only uses objective function value of each point. Also, the genetic algorithm can be applied to any continuous or discrete objective function, and in each step it surveys a set of possible points, therefore it can obtain more than one optimized response.

Here the simple genetic algorithm that has three operators namely crossover, reproduction and mutation, is used for optimization. Chromosomes in this kind of genetic algorithm has genes that their values can be zero or one (binary). The profile of the convergent section of that nozzle, which is modeled by a $2^{\text {nd }}$ order polynomial, is assumed constant. The profile of the divergent section is modeled by a $3^{\text {rd }}$ order polynomial given by

$$
y=a_{1} x^{3}+a_{2} x^{2}+a_{3} x+a_{4}
$$

To produce different shapes of this divergent section, it is sufficient to change coefficients $a_{1}$ to $a_{4}$ in their acceptable ranges (to satisfy the geometric constraints). Geometric constraints in this problem are constant throat and exit areas and the slope of the nozzle profile in throat is zero. By applying these conditions to equation (1), three coefficients of $a_{1}$ to $a_{4}$ are dependent on the others. Here only the coefficient $a_{2}$ is assumed independent and can be changed in an acceptable range for producing reasonable shapes. This coefficient changes to binary code and produces the required chromosomes for this genetic algorithm. In each generation different shapes are produced and in fact each chromosome introduces a specific shape for nozzle. First generation is produced randomly. Usually this procedure guarantees finding of a near optimum point of solution. First generation is produced by using mutation operator, which is applied on a random bit of the chromosome. The fitness evaluation is the basis of genetic algorithm and it has a great role in its selection procedures. 
The genetic algorithm recognizes chromosomes that have higher fitness values and selects them as parents for producing the next generation. In this research, finding the shape of a nozzle which is produced maximum thrust force is the goal of optimization procedure. Hence the thrust force of the nozzle is used as fitness value (objective function value) in this algorithm.

CFD software calculates thrust force of the nozzle and then transfers it to the genetic algorithm, so by this way the genetic algorithm gets objective function value. CFD software for a chromosome in each generation should be called at least once. Therefore so many calls of CFD solver are needed for a complete optimization. The numerical experiences show that time duration of numerical computation of available operations in the genetic algorithm in comparison with operations of CFD software is much small. The selection of parent is based on Roulette wheel and the probability that a parent is selected depends on its fitness value. Each pair of parents produces two offspring (two new chromosomes) by using crossover operator. Then mutation operator is applied to offspring in basis of its probability. In this research, one-point crossover is used. The crossover point which is on the parent chromosome is selected randomly. The mutation operator is performed on a gene in a chromosome which is selected randomly, and changes its value (if it is zero, it will become one and vice versa). Although the reproduction and crossover procedures are done randomly on the basis of fitness value, they may destruct valuable strings, therefore the genetic algorithm can not converge to the global optimum of problem uniformly. To have a uniform convergence, the best chromosome of each generation can be transferred to the next generation. This operator is named elitism [8]. Here, elitism is used for increasing the convergence rate and getting a uniform convergence.

\section{COMPUTATIONAL FLUID DYNAMICS (CFD)}

The run time of the objective function which is used in genetic algorithm for optimization, has a strong effect on convergence rate of genetic algorithm and on the efficiency of the genetic algorithm in optimization problems [9]. If the run time of objective function decreases, the required time of optimization will be decreased, and genetic algorithm efficiency for optimization will be increased. Hence, the objective function is a CFD software which can solve the internal flow of the nozzle and can calculate its thrust force for reducing the run time of the CFD software, the viscous flow of the nozzle is assumed quasi-one dimensional and therefore a completely uniform and quasi-one dimensional grid is produced for solving the internal flow of the nozzle.

In this research, using any kind of initial condition is possible unless it causes the divergence of the solution. Here, the uniform initial condition is used [10]. For applying the boundary condition, two kinds of boundary condition, inlet and outlet conditions, are used. By using characteristics theory, for inlet condition two or three data (based on supersonic or subsonic inlet flow) and for outlet condition zero or one datum (based on supersonic or subsonic outlet flow) are applied [11]. The governing equation of a quasi-one dimensional, viscous, and compressible flow in differential form is

$$
\frac{\partial \vec{U}}{\partial t}+\frac{\partial \vec{F}}{\partial x}=\vec{Q}
$$


where $\vec{U}$ is conservative parameters vector, $\vec{F}$ is flux vector and $\vec{Q}$ is a vector that shows the influence of quasi-one dimensionality and viscosity of the nozzle flow. These vectors are defined by equation (3)

$$
\vec{U}=\left\{\begin{array}{l}
\rho \\
\rho u \\
\rho e
\end{array}\right\} \quad \vec{F}=\left\{\begin{array}{c}
\rho u \\
\rho u^{2}+p \\
u(\rho e+p)
\end{array}\right\}, \quad \vec{Q}=-\frac{\rho u}{S(x)} \cdot \frac{d S(x)}{d x}\left\{\begin{array}{c}
1 \\
u \\
\frac{u^{2}}{2}+\frac{c^{2}}{\gamma-1}
\end{array}\right\}+\left\{\begin{array}{c}
0 \\
-\tau_{w} A_{\text {cell }} \\
0
\end{array}\right\}
$$

The first term of vector $\vec{Q}$ is the effect of one-dimensionality of the nozzle flow in which $S(x)$ is the cross section area of the nozzle, $\frac{d S(x)}{d x}$ is the cross section area change of the nozzle in $x$-direction, and $c$ is the speed of the sound. The second term of vector $\vec{Q}$ is the viscous effect of the nozzle flow, where $\tau_{w}$ is the shear stress on the walls of a cell, and $A_{\text {cell }}$ is the surface area of the cell in the direction of the nozzle flow. By assuming steady and laminar flow of the nozzle, the shear stress on the walls of each cell is calculated by considering the method which is described in reference [12]. The governing equation of internal flow of the nozzle becomes

$$
U_{i}^{n+1}=U_{i}^{n}-\frac{\Delta t}{\Delta V}\left(f_{i+\frac{1}{2}}^{(*)}-f_{i-\frac{1}{2}}^{(*)}\right)^{n}+\Delta t Q_{i}^{n}
$$

Where $f^{(*)}$ is the calculated flux on each face of a cell. The superscript $n$ determines the values of different parameters in $n \Delta t$ moment. In the first order Roe's approximation [13], the flux of each face is calculated by the equation (5)

$$
f_{i+\frac{1}{2}}^{(*)}=\frac{1}{2}\left(f_{i}+f_{i+1}\right)-\frac{1}{2} \sum_{j}\left|\bar{\lambda}_{j}\right| \partial w_{j} \vec{r}_{j}
$$

Where $\bar{\lambda}_{j}$ and $\vec{r}_{j}$ are eigenvalue and eigenvector of jacobian matrix, respectively. Also $\partial w_{j}$ determines the wave amplitude on each face of cells [11].

Here the numerical method includes a finite volume method using first order Roe's flux approximation and a time marching (explicit) method [13]. Therefore the accuracy of this method is first order. In Roe's numerical, when the value of a characteristic near the sonic line approaches to zero, the expansion waves appear which reduce the accuracy of this method. The different entropy conditions are presented for solving this problem in the Roe's method. In this research, Van leer's entropy condition is used [11]. The pure decreasing of residual value versus iteration explains the complete convergence of CFD code and reliability.

\section{GENETIC ALGORITHM VERIFICATION}

In this research for verification of the genetic algorithm application, it is used for finding the maximum of a $10^{\text {th }}$ order which is normalized by its global maximum and in the interval of $[0,1073741824]$. With respect to reference [7], the population size in each generation and chromosome length are 30 and 30, respectively. Hence the total 
number of inputs of this function becomes 1.07 billion $\left(2^{30}-1=1.07 \times 10^{9}\right)$. The crossover probability of 0.6 and the mutation probability of 0.0333 are considered. This algorithm can converge to the maximum of this function only after passing 30 generations. As the number of chromosomes in the mating pool is equal to population size of each generation, therefore this algorithm can find the maximum of that function only by 900 calls of the objective function. This number of objective function calls includes approximately $0.0001 \%$ of the total number of available points $\left(\frac{900}{1.07 \times 10^{9}} \times 100 \cong 0.0001\right)$. Fig.1 shows the growing of the maximum value and changes of the mean value of the objective function value in each generation. The robustness of the genetic algorithm which is used in this research is verified by comparing its results by reference [7].

\section{VERIFICATION OF THE CFD SOFTWARE}

The CFD software which is written by $\mathrm{C}++$ Programming language can solve two kinds of the nozzle flow, a fully supersonic flow and a flow with the subsonic inlet and outlet and a normal shock in the divergent section of the nozzle. Here the governing equations are acceptable for a quasi-one dimensional and viscous flow which its analytical solution does not exist, but the analytical solution of a quasi-one dimensional and inviscid flow is available [14]. Comparing the numerical solution of the viscous flow with the analytical solution of inviscid flow [14], shows completely identical trends for all kinds of flow parameters along the nozzle length, but the difference of their values never omits because of viscosity. After fining the grid from 50 cells to 100 , and from 100 to 200 cells, the same results are obtained and they converge to identical values. Converging the obtained results from several grids to the same values, shows the capability and robustness of this CFD software.

\section{RESULTS}

In this part, the nozzle length is assumed 10 times of the diameter of the throat, the ratio of inlet area to throat area and the ratio of the outlet area to throat area are assumed 2.5 and 12, respectively for studying of the effects of change of the genetic algorithm parameters on the internal flow of the nozzle with two kinds of boundary condition. The effects of two kinds of boundary condition (a fully supersonic flow, and a transonic flow with a normal shock wave in the divergent section of the nozzle). In the first condition, the stagnation pressure, the stagnation temperature and the inlet Mach number are assumed $100 \mathrm{kpa}, 298 \mathrm{~K}, 2.6$, respectively. In the second condition, the inlet stagnation pressure, the inlet stagnation temperature and the static outlet pressure are assumed $155 \mathrm{kpa}, 456 \mathrm{~K}$, and $100 \mathrm{kpa}$, respectively. The chromosome length is 18 bits and the selected range for changing of coefficient $a_{2}$ is the interval of $[-1.35,4.00]$. The reason for selecting this interval is the production of reasonable and acceptable shapes for the divergent section of the nozzle. With respect to this selected interval and the chromosome length, the minimum and possible increment for changing of coefficient $a_{2}$ is 0.00002 . 


\section{The Effect Change of Population Size}

The population size of each generation increases the variety in each generation, and decreases the probability of the fast convergence of the genetic algorithm to a local optimum point, but increases the convergence time of the genetic algorithm. Therefore assuming a fairly low and reasonable population size is necessary for obtaining the maximum convergence speed of the genetic algorithm. By regarding to reference [15], assuming the population size of 4 in each generation is effective on decreasing of CFD code runs, and on increasing of the convergence speed. To be optimum is the reason for choosing this value for the population size [15]. Fig.2 shows the growth trend of maximum thrust force of the nozzle with fully supersonic flow, in 30 generations and for two population sizes of 4 and 18. The comparison of these two graphs shows that the increase of population size in each generation decreases he convergence speed and does not have any effect on the accuracy of the obtained optimum point. In this research, the population size of each generation always is assumed 4 which is optimum and constant.

\section{The Effect of Using Elitism}

As the reproduction and crossover processes perform randomly, and being proportional to the fitness value, it is possible destructing the strings with high fitness value. This issue can cause that the genetic algorithm does not converge to the global optimum point uniformly. For having a uniform convergence of the genetic algorithm, the best individual of each generation (which has the highest fitness value in that generation) is transferred to the next generation. In Fig.3 the convergence trend of maximum thrust force of the nozzle with the fully supersonic flow is shown by using and without using elitism in 25 generations. The genetic algorithm by using elitism will have a uniform convergence and without using elitism its results may have fluctuation and even the genetic algorithm can not converge. Hence using the elitism is necessary for guaranteeing the convergence of the genetic algorithm.

\section{The Effect of Change of Crossover and Mutation Probabilities}

To find the optimum combination of mutation and crossover probabilities which cause obtaining of the maximum convergence speed and the minimum required time of optimization, the improvement of the genetic algorithm parameters is necessary. Only some references such as [3,7] indicated that assuming a fairly high crossover probability and a fairly low mutation probability is considered. The range of change of the crossover probability is assumed between 0.7 and 1.0 and the range of change of the mutation probability is assumed between 0.1 and 0.4 . With respect to reference [15] robustness and convergence of the genetic algorithm is guaranteed with any combination of crossover and mutation probabilities, therefore the convergence speed becomes the only important issue.

In Fig.4 the graph of the required generation for convergence is shown versus the crossover probability for constant mutation probability of 0.2 and for a fully supersonic flow. Fig.5 shows the same graph for a transonic flow with a normal shock in the divergent section of the nozzle. In this part, the mutation probability of 0.2 is chosen randomly in the interval of $[0.1,0.4]$. With respect to figures (4) and (5), it determines that the optimum value of crossover probability is independent from the type of boundary conditions, and in that range which references $[3,7]$ were determined, 
there is always an optimum crossover probability which decreases the required generations for optimization to the possible minimum value. The optimum value of this parameter is approximately equal to 0.9 for both flows.

With respect to references $[3,7]$ the mutation probability is given between 0.1 and 0.4 , of course the crossover probability value is equal to its optimum value that is 0.9 . In Fig. 6 the required number of generation for convergence is shown versus different mutation probability values for the fully supersonic internal flow of the nozzle. The optimum point of this graph becomes approximately 0.2 .

Fig.7 shows the same trend for a transonic flow with a normal shock wave which is standing in the divergent section of the nozzle. The optimum point of this graph is approximately 0.3 .

In these both cases, the value of crossover probability is 0.9 which is the optimum value. For verification of the obtained optimum values of crossover and mutation probabilities which are calculated for the first boundary condition (a fully supersonic flow) 0.9 and 0.2 , and for the second boundary condition (a transonic flow with a normal shock wave in the divergent section of the nozzle) 0.9 and 0.3 , respectively, a crossover probability is selected randomly and the optimization procedure is performed by changing mutation probability in its determined range.

Finally results of this case are compared with the results of the optimum case. This random crossover probability is assumed 0.7. In Fig.8 the graph of the required number of generations for convergence is sketched versus mutation probability. These graphs are for two different crossover probabilities of 0.7 and 0.9 , and the internal flow of the nozzle in this case is a fully supersonic one. With respect to Fig.8 simultaneous usage of the crossover probability of 0.9 and mutation probability of 0.2 the highest convergence speed among other combinations of these two parameters. Therefore the improved values of the genetic algorithm parameters that is the optimum crossover and mutation probabilities, which maximize the convergence speed of the genetic algorithm for a fully supersonic nozzle flow, will be equal to 0.9 and 0.2 , respectively.

Fig.9 shows the changing trend of convergence speed versus mutation probability. Here these two graphs are sketched for two different crossover probabilities of 0.7 and 0.9 , and the internal flow of the nozzle is a transonic one with a normal shock wave in the divergent section of the nozzle. In this case the combination of crossover probability of 0.9 and mutation probability of 0.3 produces the maximum convergence speed for the genetic algorithm. Hence the improved values of the genetic algorithm parameters for a transonic nozzle flow which has a normal shock wave in the divergent section of the nozzle are 0.9 for the crossover probability 0.3 for the mutation probability.

Therefore the improved genetic algorithm with crossover probability of 0.9 and mutation probability of 0.2 for a fully supersonic flow, and with crossover probability of 0.9 and mutation probability of 0.3 for a transonic flow which has a normal shock wave in the divergent section of the nozzle, can optimize the shape of a convergentdivergent nozzle for obtaining the maximum thrust force with minimum CFD code calls.

The two graphs of Fig.10 show the maximum thrust force of the nozzle with a fully supersonic flow in 60 generation, once with using the improved genetic algorithm and once without using random crossover and mutation probabilities. The improved crossover and mutation probabilities for this flow are 0.9 and 0.2 and random values are assumed 0.6 and 0.02 respectively. In Fig.11 the value of coefficient $a_{2}$ is 
sketched in 60 generations for this case. By regarding to figures (10) and (11) the improved genetic algorithm parameters increase the convergence speed of the genetic algorithm, also increase the accuracy of the obtained results.

The two graphs of Fig.12 show the maximum thrust force of the nozzle with a transonic nozzle flow which has a normal shock wave in the divergent section of the nozzle in 60 generation, once with using the improved genetic algorithm and once without using random crossover and mutation probabilities. The improved crossover and mutation probabilities for this flow are 0.9 and 0.3 , and random values are assumed 0.6 and 0.02 respectively. In Fig.13 the value of coefficient $a_{2}$ is sketched in 60 generations for this case. By regarding to figures (12) and (13) the improved genetic algorithm parameters increase the convergence speed of the genetic algorithm, also increase the accuracy of the obtained results.

In all cases which are discussed, the genetic algorithm can find the optimum point of the problem after passing 60 generations (at maximum) with constant population size of 4 . As the population size of each generation is equal to the population size of the mating pool, therefore this algorithm calls the CFD code 240 times. This number of CFD calls includes only $0.09 \%\left(\frac{240}{2^{18}-1} \times 100 \cong 0.09\right)$ of all possible CFD code calls.

This issue determines the robustness and efficiency of the genetic algorithm in optimization of shape of convergent-divergent nozzles for getting maximum thrust force.

The optimum value of the coefficient $a_{2}$ which is the controlling parameter of the shape of the divergent section of the nozzle for both types of flow (a fully supersonic flow or a transonic flow with a normal shock wave in the divergent section of the nozzle) is equal to 3.98 , and values of other coefficients of equation (1) namely $a_{1}$, $a_{3}$, and $a_{4}$ which are dependent to $a_{2}$, become $-0.177,-26.525$, and 56.25, respectively. By regarding to the values of these coefficients, the slope of the divergent section of the nozzle at the exit becomes zero. In Fig.14 the optimum shape of the nozzle which is obtained by the genetic algorithm for getting maximum thrust force is sketched.

\section{CONCLUSION}

Here capability and efficiency of the genetic algorithm is studied in optimization of the shape of convergent-divergent nozzles for getting maximum thrust force. The genetic algorithm which is used here, is simple and has three operators namely reproduction, crossover and mutation, and also uses the elitism. The CFD code which is used for solving the internal flow of the nozzle and for calculating the nozzle thrust force uses a finite volume method using the first order Roe's Approximation and time marching (explicit) method. The capability or robustness of this genetic algorithm is shown by finding the similar shapes for different combinations of population size, crossover and mutation probabilities. With respect to performed comparisons for different genetic algorithm parameters, the efficiency and convergence speed of the genetic algorithm is highly increased after improving these parameters. By using these optimum parameters and using elitism, the optimum shape of the nozzle is found with only less than $0.1 \%$ of the total number of possible CFD code calls. This low number of CFD 
code calls increases the efficiency and accuracy of the genetic algorithm which is used in optimization of the shape of convergent-divergent nozzles for getting maximum thrust force.

\section{FIGURES}

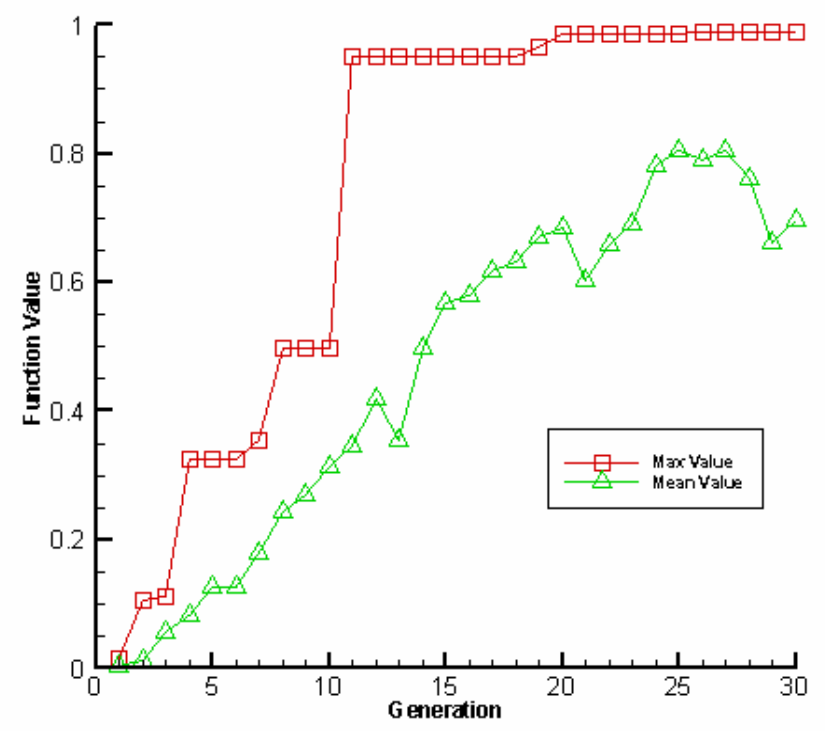

Fig.1.The maximum and mean values of $10^{\text {th }}$ order polynomial in each generation

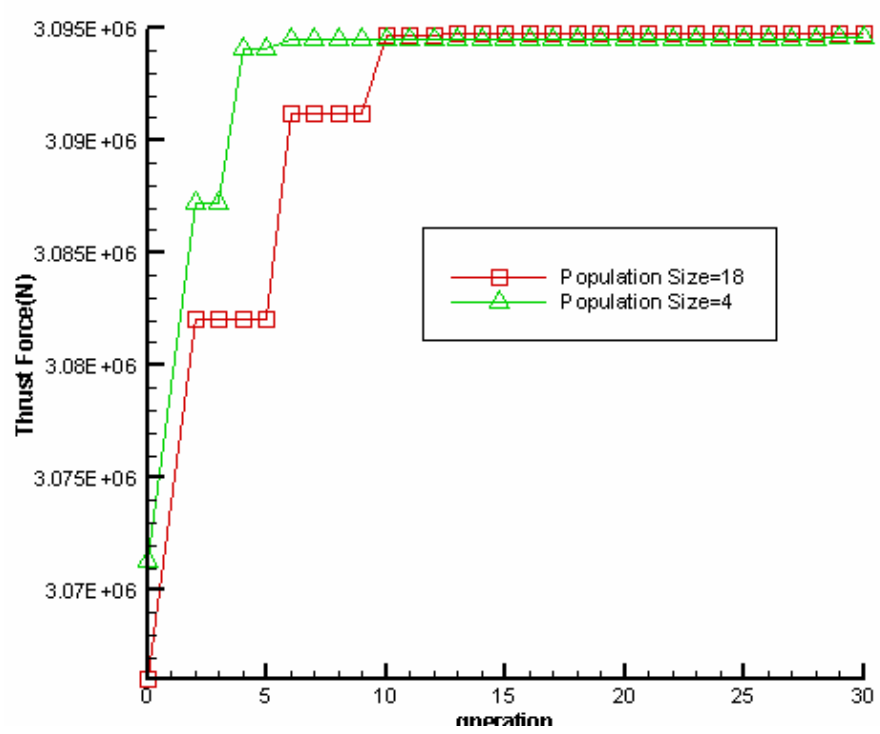

Fig.2.The maximum thrust force for population sizes of 4 and 18 in each generation (fully supersonic flow) 


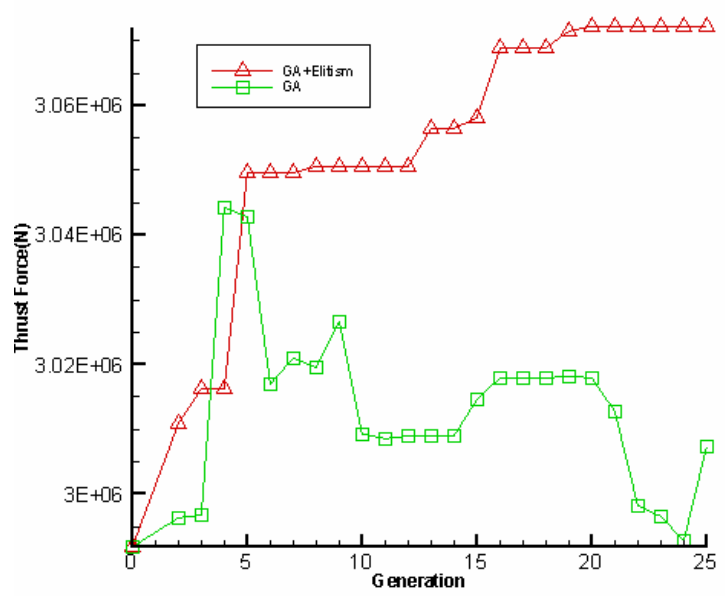

Fig.3. Comparison of growth trends of the maximum thrust force of the nozzle between using and without using elitism

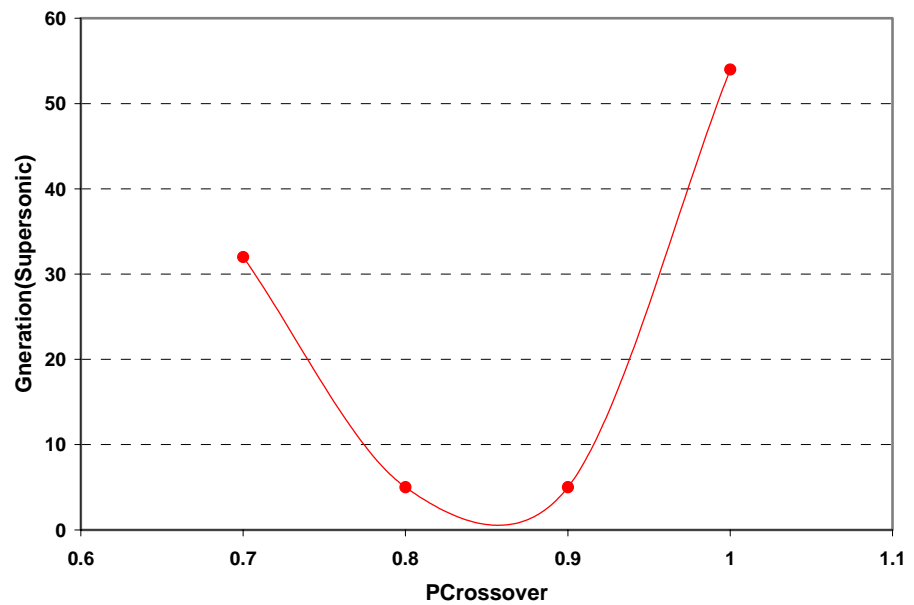

Fig.4. The required number of generations for convergence versus crossover probability (fully super sonic flow)

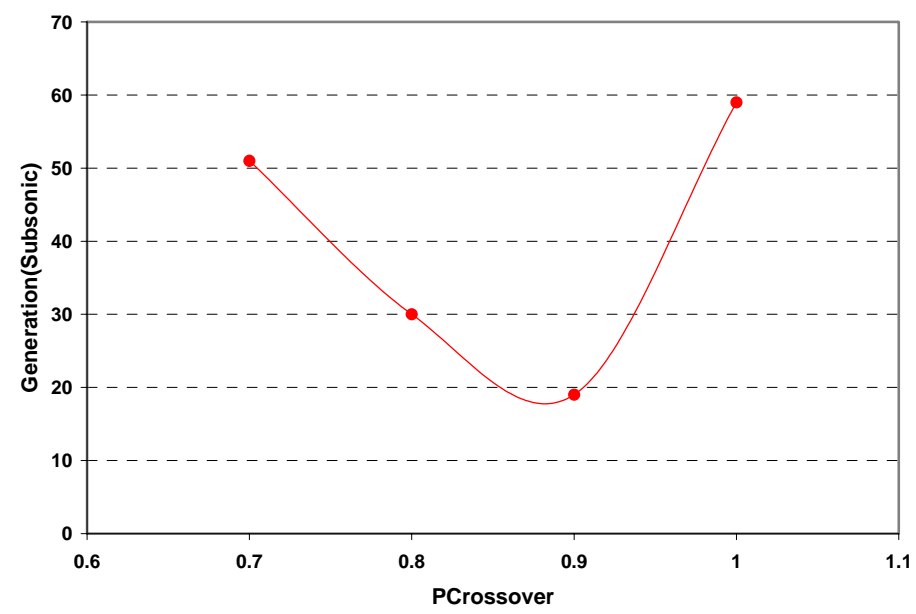

Fig.5. The required number of generations for convergence versus crossover probability (transonic flow with a normal shock) 


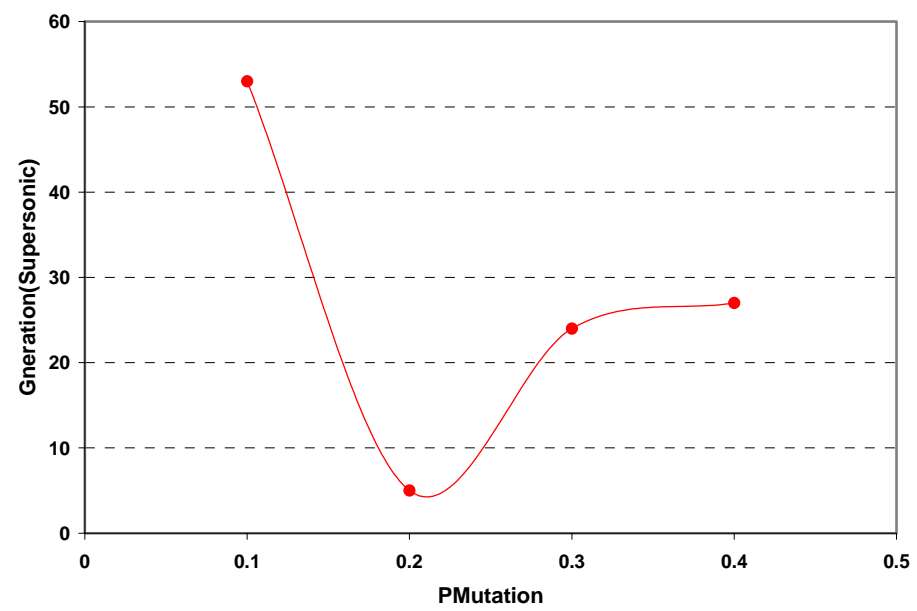

Fig.6. The required number of generations for convergence versus mutation probability (fully super sonic flow)

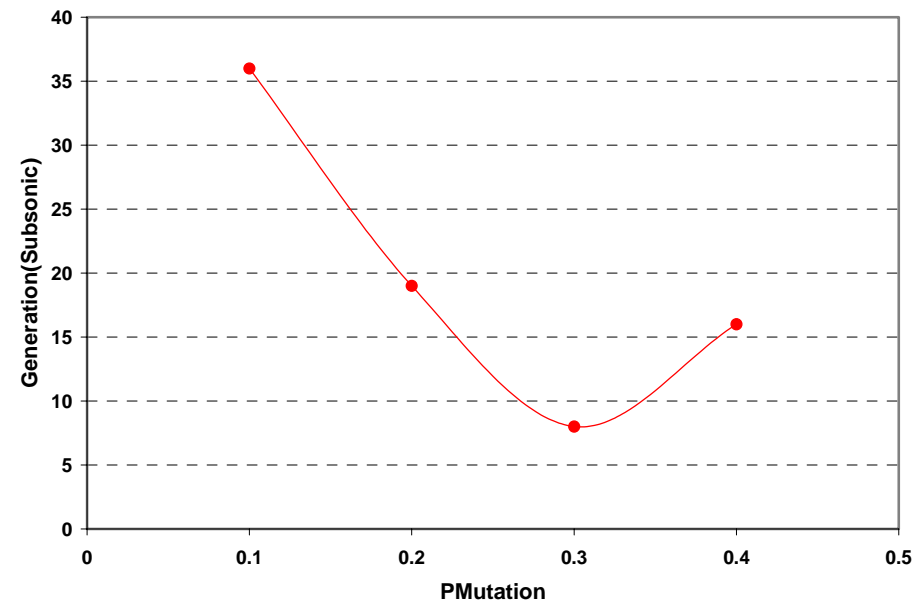

Fig.7. The required number of generations for convergence versus mutation probability (transonic flow with a normal shock)

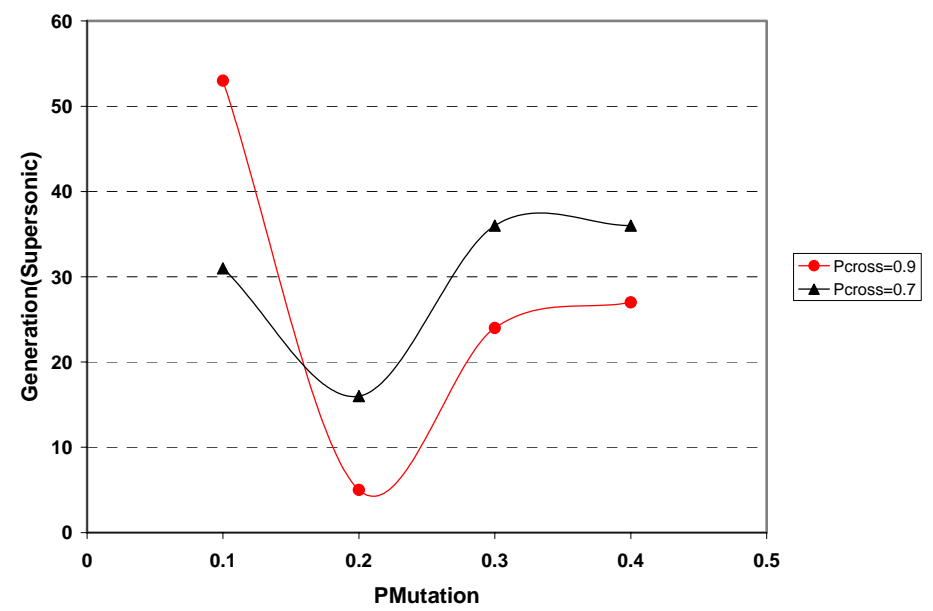

Fig.8. The required number of generations for convergence versus mutation probability of a fully super sonic flow by using two different crossover probabilities of 0.7 and 0.9 . 


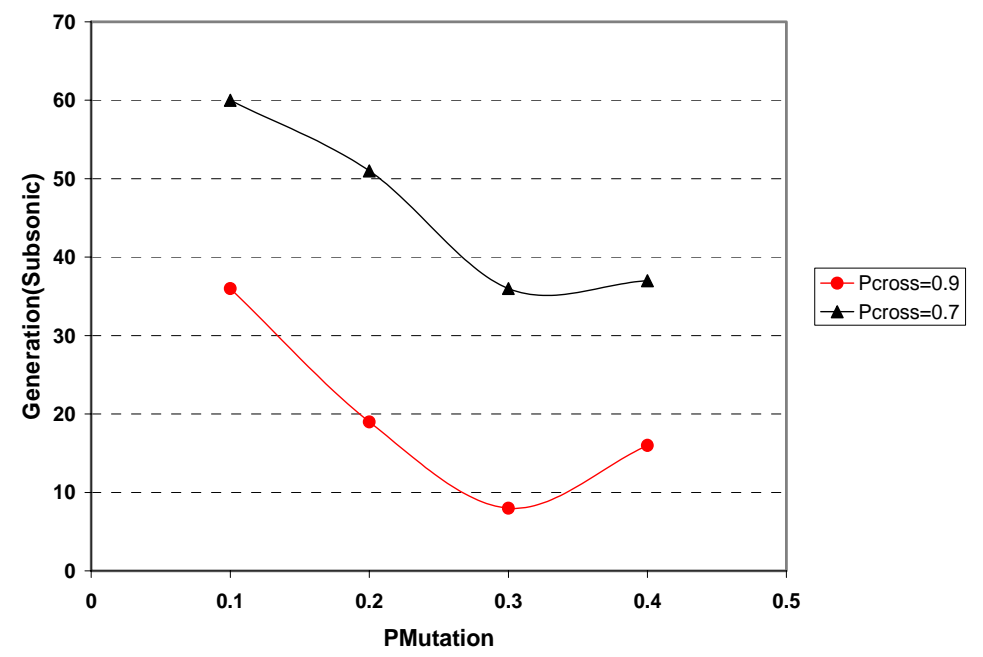

Fig.9. The required number of generations for convergence versus mutation probability of a transonic flow by using two different crossover probabilities of 0.7 and 0.9

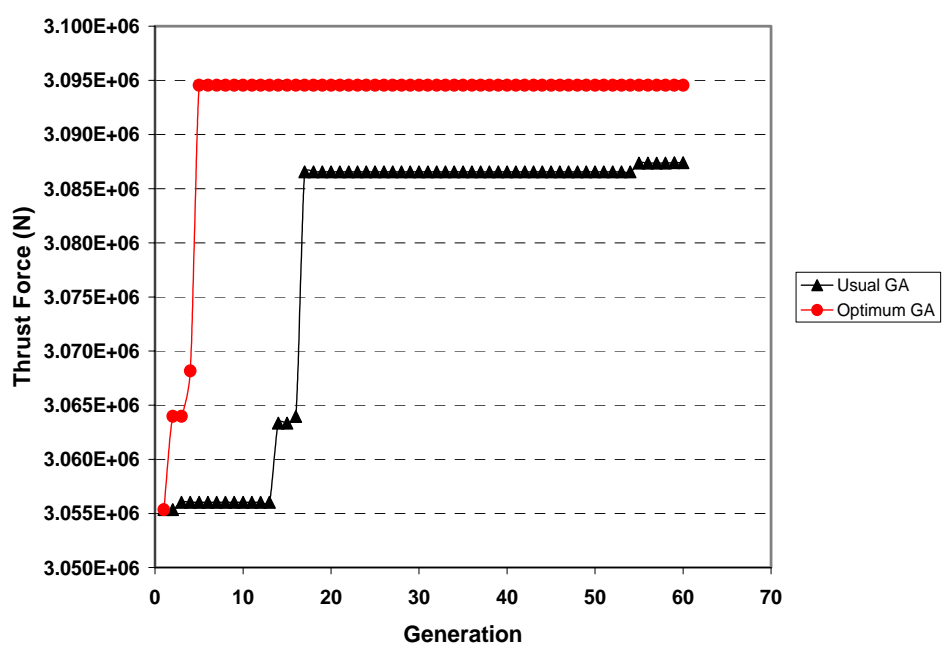

Fig.10. Comparison of growth trends of maximum thrust force for a fully supersonic flow using normal and optimum genetic algorithms.

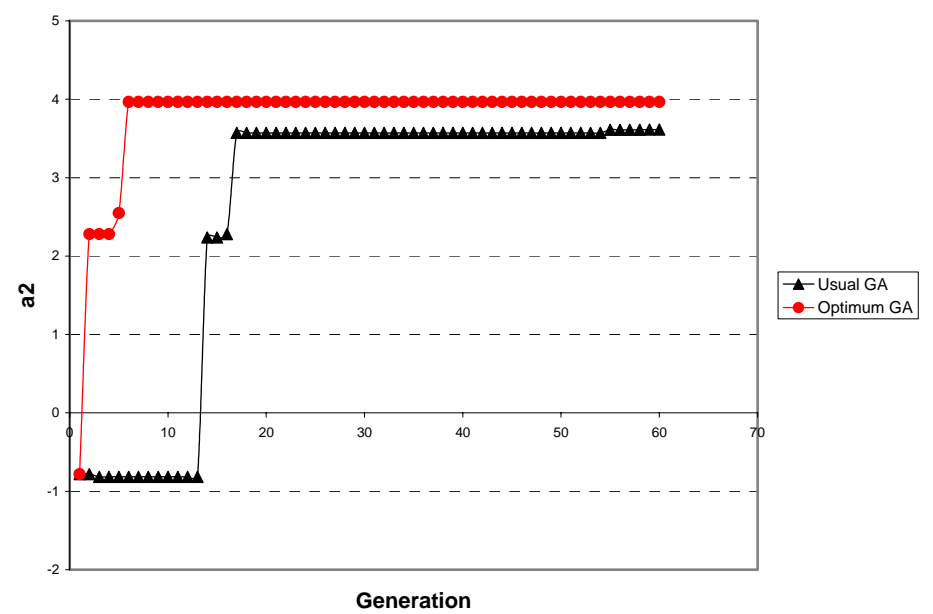

Fig.11. Comparison of growth trends of coefficient a2 for a transonic flow by using normal and optimum genetic algorithms. 


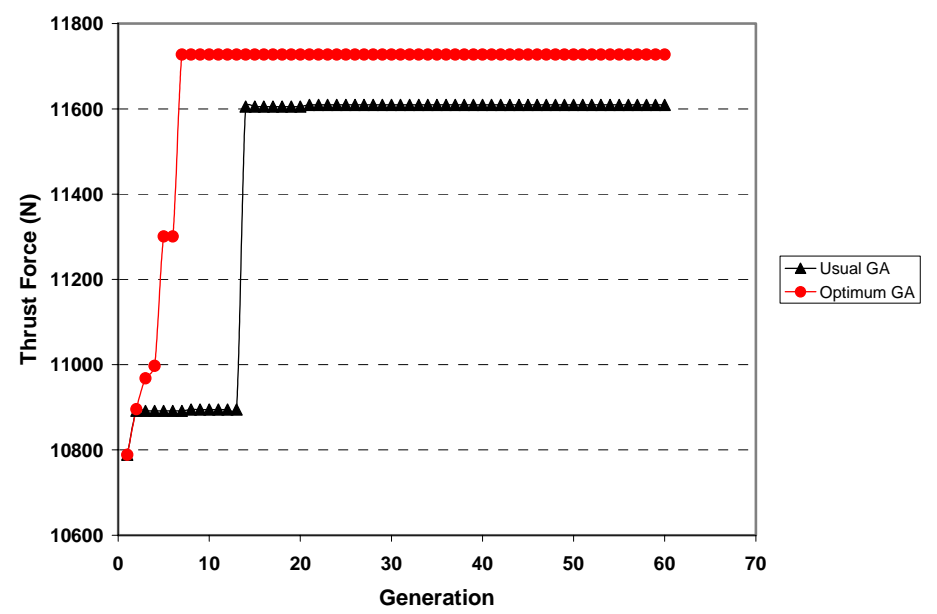

Fig.12. Comparison of growth trends of maximum thrust force for a transonic flow using normal and optimum genetic algorithms.

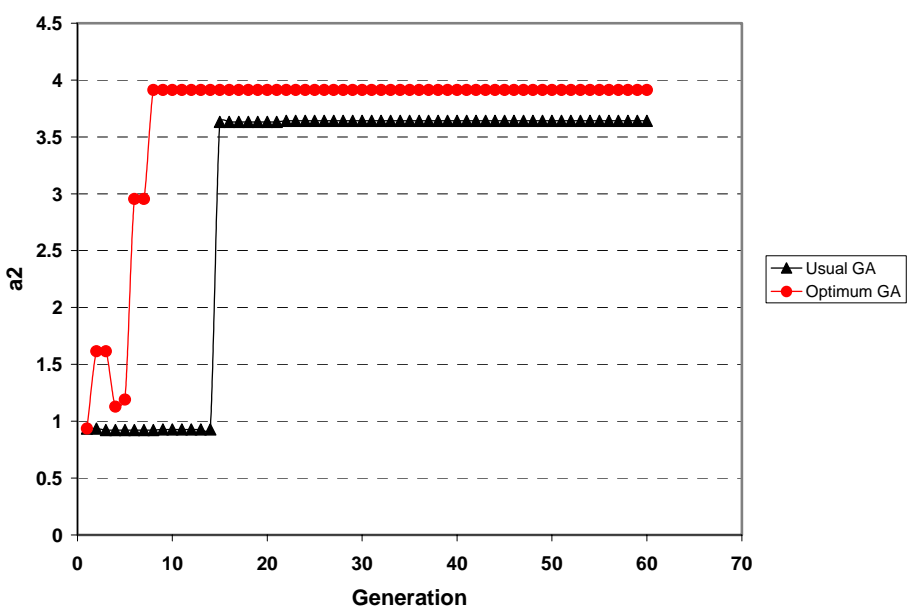

Fig.13. Comparison of growth trends of coefficient a2 for a transonic flow by using normal and optimum genetic algorithms.

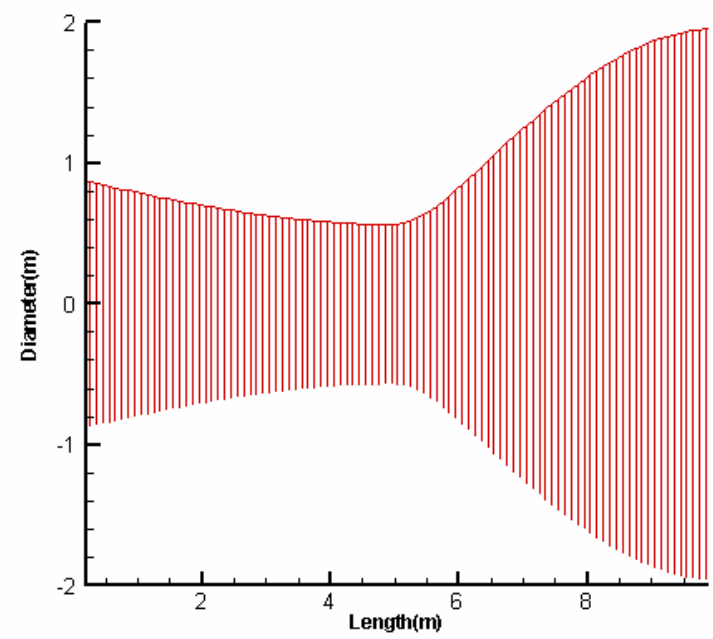

Fig.14. The optimum shape of the nozzle for two different boundary conditions 


\section{REFERENCES}

[1] Obayashi, S. and Tsukahara,T., "Comparison of Optimization Algorithm for Aerodynamic Shape Design", AIAA paper 96-2304-cp, 1996.

[2] Quagliaerlla, D. and Cioppa, A. D., "Genetic Algorithms Applied to the Aerodynamic Design of Transonic Airfoils", AIAA paper, 94-1896, 1996.

[3] Quagliaerlla, D., "Genetic Algorithms Applications in Computational Fluid Dynamics", $2^{\text {nd }}$ edition, John Wiley, New York, 1995.

[4] Lynch, F., "Commercial Transports Aerodynamic Design for Cruise Performance Efficiency", $1^{\text {st }}$ edition, Nixon, New York, 1982.

[5] Chan, Y., "Application of Genetic Algorithms to Aerodynamic Design", Canadian Aeronautics and Space Journal, Vol. 44, No.3, 1998, pp. 182-187.

[6] Dastagupta, D., Michalewicz, Z., "Evolutionary Algorithms in Engineering Application", Springer, 1997.

[7] Goldberg, D.. "Genetic Algorithms in Search, Optimization, and Machine Learning", $2^{\text {nd }}$ edition, Addison-Wesley, 1989.

[8] Davis, L., "Handbook of Genetic Algorithm", $1^{\text {st }}$ edition, Van Nostrand Reinhold, New York, 1990.

[9] Quagliaerlla, D.," Genetic Algorithms in Engineering and Computer Science", John Wiley, New York, 1995.

[10] Khaksarfard, R., "Convergence Acceleration of a Navier-Stokes Solver by Agglomeration Multigrid", M.Sc. Thesis, Aerosoace Eng. Dep., Sharif University of Technology, January 2006.

[11] Hirsch, C., "Numerical Computation of internal and External Flows", $1^{\text {st }}$ edition , Vol. 2, John Wiely \& Sons, 1990.

[12] Shapiro, A. H.," The Dynamics and Thermodynamics of Compressible Fluid Flow", Vol. 1, John Wiely \& Sons, 1953.

[13] Roe, L.," Approximate Riemann Solvers", Journal of Computational Physics, Vol. 43, 1981, pp. 357-372.

[14] Anderson, J., "Modern Compressible Flow", $1^{\text {st }}$ edition, McGraw-Hill Book Company, 1982.

[15] Khayatzadeh P., Mazaheri K., "Shape Optimization Of Nozzles By Means Of Genetic Algorithms For Getting Maximum Thrust Force", AERO2007 conference (Iranian Aerospace Engineering Society) on Feb. 24-26, 2007, K.N. Toosi university of Technology, Tehran, Iran. 